Reprod. Nutr. Develop., 1988, 28 (3 B), 831-837

\title{
La calpaine II du muscle squelettique de lapin. Régulation de l'activité enzymatique: influence de la présence de phospholipides membranaires sur l'autolyse enzymatique en présence ou non de substrat
}

\author{
P. COTTIN, Ch. GARRET, A. DUCASTAING
}

Laboratoire de Biochimie et Technologie des Aliments

Université de Bordeaux I, Avenue des Facultés, 33045 Talence Cedex, France.

Summary. Calpain // in rabbit skeletal muscle. Enzymatic activity regulation : incidence of membrane phospholipids on the enzymatic autolysis in the presence or not of substrate.

A calcium activated neutral proteinase (calpain II) was prepared on a large scale at a high degree of purity from rabbit skeletal muscle using an original purification procedure

« / $n$ vitro» highly purified Calpain II was optimally activated by $2 \mathrm{mM} \mathrm{Ca}^{2+}$ and had two subunits with a molecular weight of $80 \mathrm{kDa}$ and $28 \mathrm{kDa}$. The influence of membrane acid phospholipids (phosphatidyl inositol PI, phosphatidyl serine PS) and neutral phospholipids (phosphatidyl choline) on calcium enzymatic autolysis in the presence or not of substrate was electrophoretically studied. In the absence of a substrate no degradation of the catalytic subunit $(80 \mathrm{kDa})$ was observed : phosphatidyl inositol and phosphatidyl serine stimulated drastically the $28 \mathrm{kD}$ a subunit degradation with a release of a common $18 \mathrm{kDa}$ product. In the presence of substrate ( $\beta$-globin) the $28 \mathrm{kDa}$ subunit was more protected against calcium autolysis. On the other hand, phosphatidyl serine seemed to induce simultaneously 80 and $28 \mathrm{kDa}$ degradations : the released breakdown products were different from those obtained with phosphatidyl inositol and phosphatidyl choline.

In presence or absence of substrate, phosphatidyl choline did not affect significantly the calpain II calcium autolysis. Further studies on calpain II-membrane binding as well as its influence on calpain -activity are in progress.

\section{Introduction.}

La protéase neutre calcium dépendante (E.C.3.4.22.17) connue sous le nom de calpaine II est une thiol protéase intracellulaire constituée de deux sous-unités de $P M=80000$ et 30000 (Dayton et al., 1976a; 1976b). Différents résultats obtenus in vitro laissent penser que cette enzyme peut participer à de nombreuses fonctions biologiques régulées par le calcium (Azanza et al., 1979; Hutson et Krebs, 1968 ; Puca et al., 1977). Expérimentalement, elle permet de reproduire sur les protéines myofibrillaires des « images " électrophorétiques analogues à celles observées au cours des phénomènes de maturation des viandes (Azanza et al., 1979, 1980 ; Merdaci et al., 1983). Cependant, le problème du rôle cellulaire est aussi lié à la régulation, car la concentration en calcium nécessaire in vitro pour une activité optimale de la calpaine II est nettement plus élevée que celle rencontrée classiquement dans la cellule musculaire. 
Des travaux récents (Hathaway et al., 1982 ; De Martino et al., 1986) ont pu montrer que des formes autolysées (en présence de calcium) de la protéase, deviennent actives pour des concentrations en calcium dix fois moins élevées. Ces phénomènes d'autolyse apparaissent directement liés à la présence de phospholipides membranaires (Coolican et Hathaway, 1984) et se traduisent essentiellement par une dégradation de la sous-unité 30000 considérée par certains auteurs (Coolican et al., 1986; Imajoh et al., 1986) comme une sous-unité régulatrice.

Nous avons donc voulu vérifier si le mécanisme d'autolyse enzymatique était une étape nécessaire à l'activation de l'enzyme d'une part et si la présence du substrat et des phospholipides modulait ou non ce comportement. Deux types de phospholipides ont été testés (phospholipides acides: phosphatidyl inositol (PI), phosphatidyl sérine (PS) phospholipide neutre: phosphatidyl choline (PC))

\section{Matériel et méthodes.}

\section{Matériel.}

Les produits chimiques utilisés proviennent de Merck (caséine, 2-mercaptoéthanol); Boehringer Mannheim S.A. (Tris); Fluka (EDTA, EGTA) ; Serva (Brillant Blue R) ; Prolabo (SDS, sodium azoture) ; Sigma (phospholipides et $\beta$ globine). L'extraction de la calpaine II a été effectuée à partir de muscles lombaires (longissimus dorsi) de lapin (fauve de Bourgogne) pesant entre 3 et $4 \mathrm{~kg}$.

\section{Méthodes.}

Mesure de l'activité enzymatique. - Les activités enzymatiques de la calpaine II ont été réalisées à partir d'un substrat protéique (caséine à $5 \mathrm{~g} / \mathrm{I}$ ) selon le protocole décrit par Azanza et al. (1979) : une unité enzymatique est définie comme étant la quantité d'enzyme qui provoque une augmentation de la densité optique à $280 \mathrm{~nm}$ de $10^{-3}$ unité par minute.

Détermination des concentrations protéiques. - Les concentrations protéiques sont déterminées selon la méthode de Bradford (1976) à partir d'une gamme étalon de sérum albumine bovine.

Analyse électrophorétique en milieu dénaturant (SDS). - La technique utilisée est proche de celle de Laemmli (1970): la migration est réalisée en gradient continu de polyacrylamide (10-20\%).

Préparation des phospholipides membranaires. - A partir de solutions mères conservées en chloroforme (95\%), éthanol (5\%), des prélèvements aux concentrations voulues sont effectués. Les solvants sont évaporés sous courant d'azote et les phospholipides repris en tampon (Tris- $\mathrm{HCl} 20 \mathrm{mM}, \mathrm{pH}=7,50$ contenant EDTA $1 \mathrm{mM}$, 2-mercaptoéthanol $14 \mathrm{mM}$ et $\mathrm{NaN}_{3} 1 \mathrm{mM}$ ) sont soumis aux ultrasons $(2 \times 4 \mathrm{~min})$ juste avant utilisation.

Préparation de l'extrait brut. — Les lapins sont sacrifiés par rupture cervicale, les muscles lombaires prélevés, coupés finement et homogénéisés par passage au 
Warring Blendor $(3 \times 30 \mathrm{sec})$ dans 3 volumes de tampon d'extraction : Tris- $\mathrm{HCl}$ $20 \mathrm{mM}, \mathrm{pH}=7,50$, EDTA $2 \mathrm{mM}$, EGTA $2 \mathrm{mM}$, 2-mercaptoéthanol $14 \mathrm{mM}$, $\mathrm{NaN}_{3} 1 \mathrm{mM}, \mathrm{NaCl} 50 \mathrm{mM}$.

La suspension obtenue est centrifugée $(30 \mathrm{~min}$ à $3000 \mathrm{~g})$, le $\mathrm{pH}$ du surnageant est ramené à $\mathrm{pH}=7,50$ par addition de Tris $1 \mathrm{M}$. Les culots sont repris pour une nouvelle extraction dans un volume de tampon, homogénéisés puis centrifugés comme précédemment. La suspension obtenue est amenée à une concentration en $\mathrm{NaCl} 1 \mathrm{M}$ par addition de chlorure de sodium solide et ajustée à $\mathrm{pH} 7,50$. Cette suspension est clarifiée par centrifugation à $12000 \mathrm{~g}$ pendant $30 \mathrm{~min}$.

\section{Résultats.}

Purification de la calpaine //.

Elle est réalisée selon une modification du protocole décrit par Penny et al. (1985) par la mise en œuvre de 4 étapes chromatographiques (une phénylSepharose, une DEAE-Sephacel, une deuxième phényl-Sepharose et une mono- $\mathrm{Q}$, HR5/5) (Garret et al., 1988).

Le protocole expérimental utilisé nous a permis d'obtenir $8 \mathrm{mg}$ de calpaine II hautement purifiée à partir de $2 \mathrm{~kg}$ de muscle squelettique. La calpaine II obtenue présente deux sous-unités correspondant respectivement à des PM de 80000 et 28000 .

Influence des phospholipides membranaires et d'un substrat ( $\beta$-globine) sur l'autolyse enzymatique (pour des concentrations en calcium de $2 \mathrm{mM}$ ).

\section{- En absence de substrat.}

Par rapport au témoin (fig. 1A), la dégradation de la sous-unité de $P M=30000$ est accélérée en présence de PI et PS. En effet, dans nos conditions expérimentales, cette dégradation apparaît quasi-totale dès $40 \mathrm{sec}$ d'incubation.

$\mathrm{Si}$ les vitesses de dégradation en présence de PI et PS semblent analogues (fig. 1B, 1C) les produits d'hydrolyse obtenus sont différents: en effet, en présence de PS (fig. 1C) trois bandes protéiques majeures sont notables ayant des PM respectifs de 24000,18000 et 8000 . Par contre, en présence de PI, on ne détecte qu'un seul produit de dégradation ( $P M=18000)$.

En présence de PC (fig. 1D) aucune différence significative par rapport au témoin n'a pu être mise en évidence, tant au niveau de la vitesse de dégradation de la sous-unité 28000 qu'au niveau du produit d'hydrolyse apparu de $\mathrm{PM}=18000$.

Quel que soit le phospholipide interagissant, aucune dégradation de la sous-unité catalytique de $\mathrm{PM}=80000$ n'a pu être mise en évidence. Ces résultats corroborent ceux obtenus par De Martino et al. (1986).

\section{- En présence de substrat.}

Les résultats (fig. 2) montrent que la vitesse de dégradation de la sous-unité 28000 en présence de PC (fig. 2 C) reste sensiblement identique à celle du 


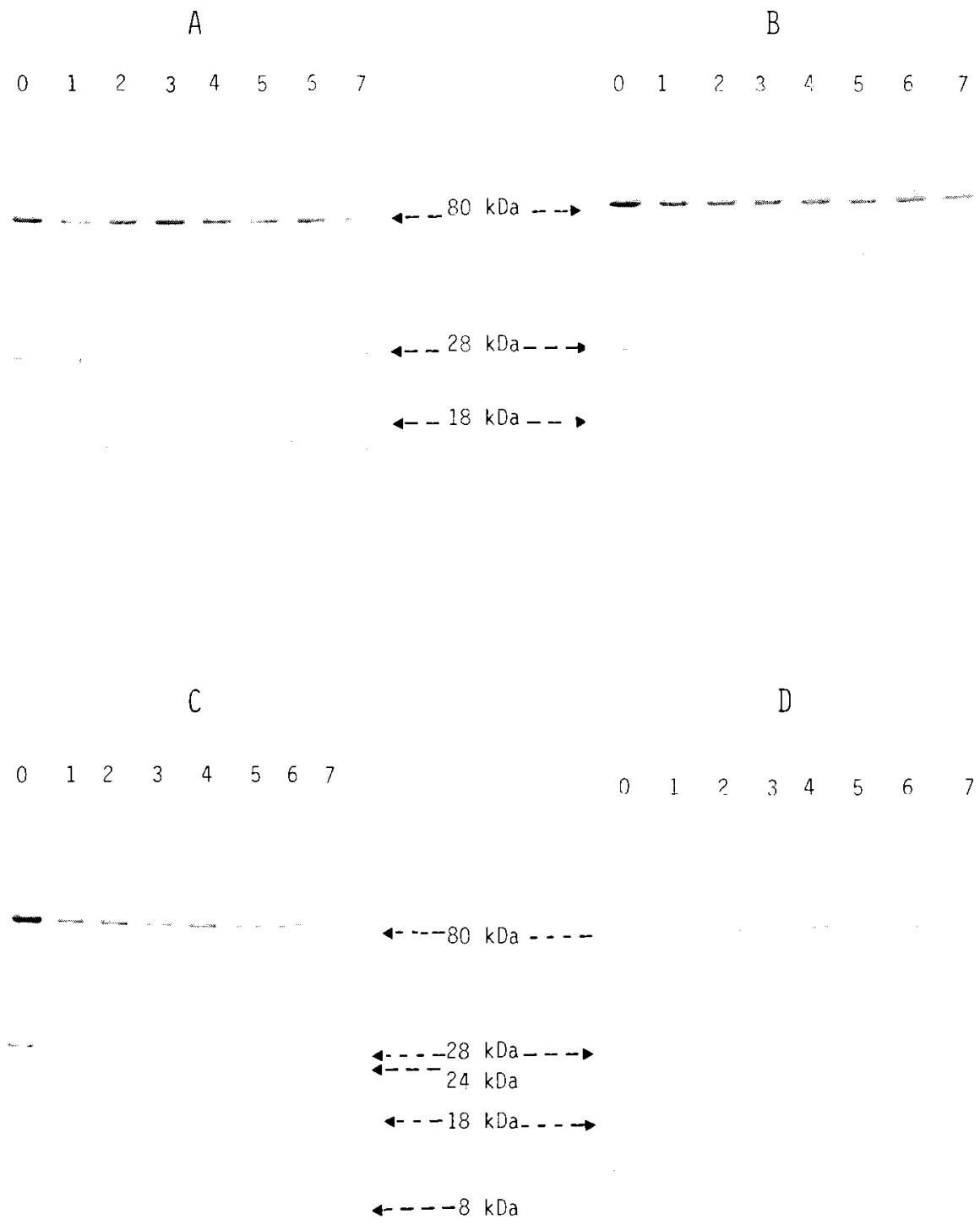

FIG. 1. - Analyse électrophorétique sur gradient de polvacrylamide (10-20\%) en milieu SDS des fractions calpaine $1 /$ incubées à $10^{\circ} \mathrm{C}$ en présence de $\mathrm{Ca}^{2+}(2 \mathrm{mM})$ pendant différents temps $(1: 20 \mathrm{~s}, 2: 40 \mathrm{~s}, 3: 80 \mathrm{~s}, 4: 120 \mathrm{~s}, 5: 5 \mathrm{~min}, 6: 8 \mathrm{~min}, 7: 15 \mathrm{~min})$.

A : calpaine II; B : calpaine II + phosphatidyl inositol; $C$ : calpaine $\|=$ phosphatidyl sérine : D : calpaine II = phosphatidyl choline.

Les rapports molaires phospholipides/calpaine II sont dans nos conditions expérimentales de 15

Des solutions de calpaine $\|(0,2 \mathrm{mg} / \mathrm{ml})$ et de phospholipides $(10 \mathrm{mg} / \mathrm{ml})$ en tampon Tris- $\mathrm{HCl}$ $20 \mathrm{mM} \mathrm{pH}=7,50$ contenant EDTA $1 \mathrm{mM}, 2$-mercaptoéthanol $14 \mathrm{mM}$ et $\mathrm{NaN}_{3} 1 \mathrm{mM}$ sont préincubées à $10^{\circ} \mathrm{C}$ pendant $5 \mathrm{~min}$. La réaction est démarrée par addition d'une solution de $\mathrm{CaCl}_{2}(10 \mathrm{mM}) .4 \mu \mathrm{g}$ de protéine ont été déposés. 
A

$\begin{array}{llllllll}0 & 1 & 2 & 3 & 4 & 5 & 6 & 7\end{array}$

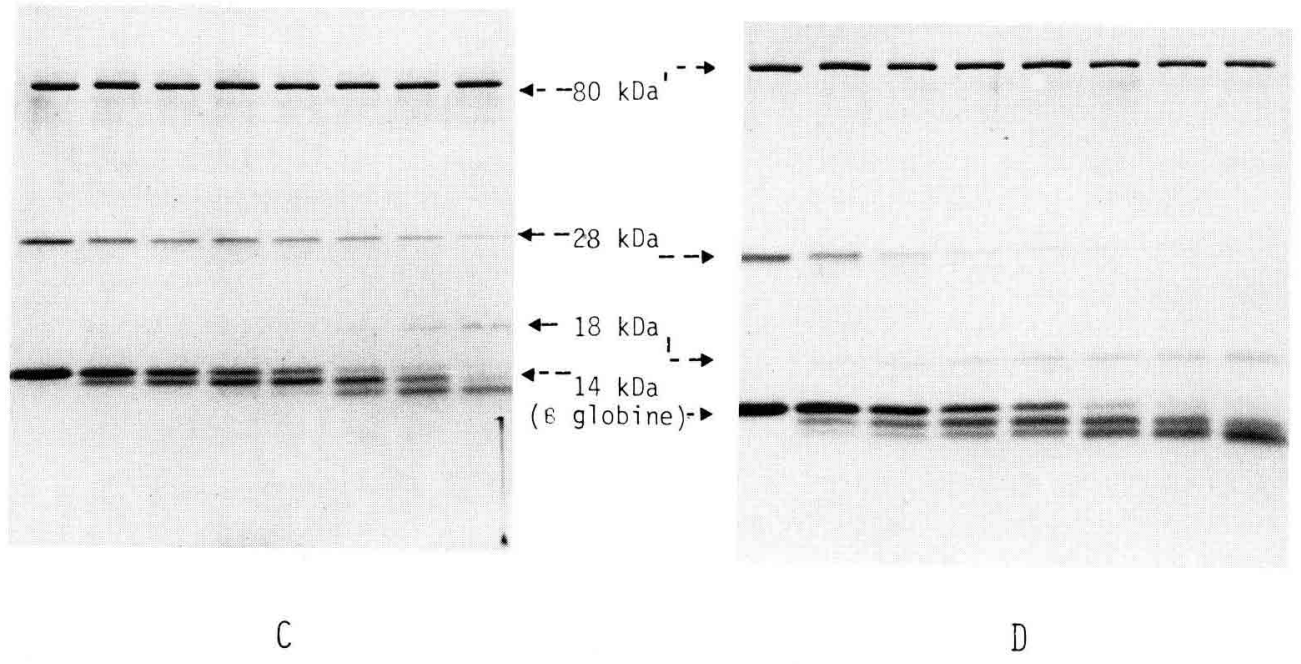

$\begin{array}{lllllll}0 & 1 & 2 & 3 & 4 & 5 & 6\end{array}$

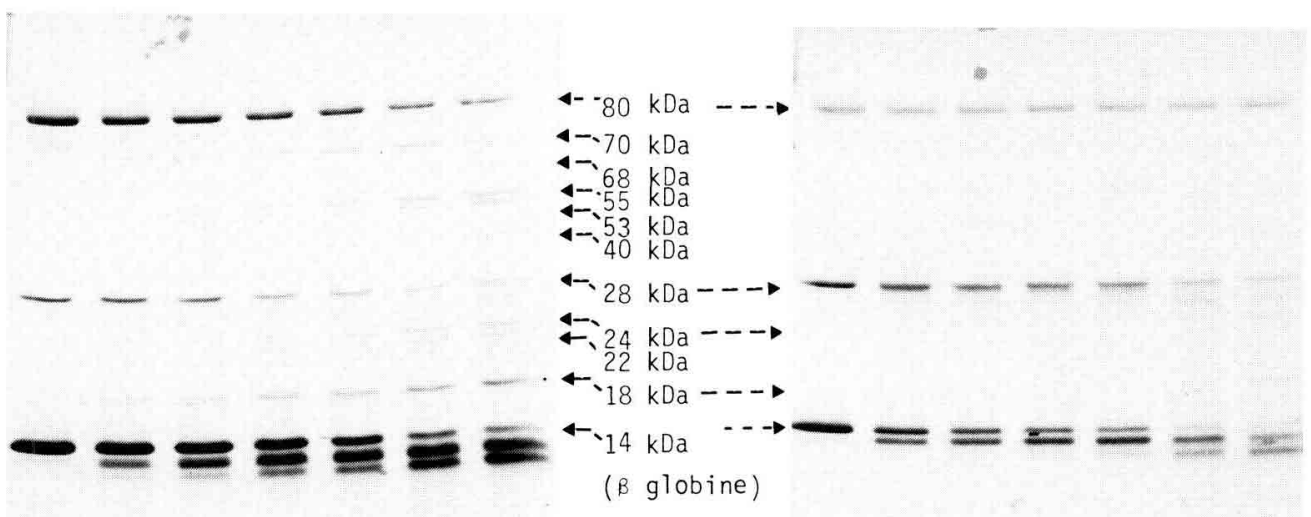

FIG. 2. - Analyse électrophorétique sur gradient de polyacrylamide (10-20\%) en milieu SDS des cinétiques d'autolyse à $10^{\circ} \mathrm{C}$ de la calpaine // en présence de substrat $\beta$-g/obine $(5 \mathrm{mg} / \mathrm{ml})$ et de phospholipides $(10 \mathrm{mg} / \mathrm{ml})$ pour une concentration en $C a^{2+}$ de $2 \mathrm{mM}$. Les temps de cinétique retenus sont : $1: 20 \mathrm{~s}, 2: 40 \mathrm{~s}, 3: 80 \mathrm{~s}, 4: 120 \mathrm{~s}, 5: 5 \mathrm{~min}, 6: 8 \mathrm{~min}, 7: 15 \mathrm{~min}$.

A : calpaine II $+\beta$-globine; $\mathrm{B}$ : calpaine II + phosphatidyl inositol $+\beta$-globine ; $\mathrm{C}$ : calpaine II + phosphatidyl sérine $+\beta$-globine; $\mathrm{D}$ : calpaine II + phosphatidyl choline $+\beta$-globine.

Le rapport calpaine $I / / \beta$-globine dans nos conditions expérimentales est $0,2(1 / 5)$. Les rapports molaires phospholipides/calpaine II sont dans nos conditions expérimentales de 15.

Des solutions de calpaine II $(0,2 \mathrm{mg} / \mathrm{ml})$ et de phospholipides $(10 \mathrm{mg} / \mathrm{ml})$ en tampon Tris- $\mathrm{HCl}$ $20 \mathrm{mM} \mathrm{pH}=7,50$ contenant EDTA $1 \mathrm{mM}$, 2-mercaptoéthanol $14 \mathrm{mM}$ et $\mathrm{NaN}_{3} 1 \mathrm{mM}$ sont préincubées à $10^{\circ} \mathrm{C}$ pendant $5 \mathrm{~min}$. La réaction est démarrée par addition d'une solution contenant la $\beta$-globine $(5 \mathrm{mg} / \mathrm{ml})$ et $\mathrm{CaCl}_{2}(10 \mathrm{mM}) .9 \mu \mathrm{g}$ de protéine ont été déposés. 
témoin (fig. 2A) alors que celle enregistrée en présence de PS et de PI augmente pour devenir maximale en présence de PI (fig. 2B, 2C). L'incubation avec PS entraîne parallèlement une dégradation très rapide de la sous-unité de $P M=80000$ dès $20 \mathrm{sec}$ d'incubation, laquelle se traduit par l'apparition de bandes protéiques ayant des PM respectifs de 70, 68, 55, 53 et 40000 (fig. 2C). Dans nos conditions expérimentales, il apparaît difficile d'attribuer les produits de dégradation de $P M=24000$ et 22000 uniquement à la sous-unité de $P M=28000$.

Quoi qu'il en soit, l'influence de PS sur l'autolyse enzymatique semble être très particulière et spécifique, affectant les deux sous-unités de manière quasi simultanée.

Par rapport au complexe calpaine II-phospholipides, la présence de «substrat » d'une manière générale semble ralentir la vitesse d'autolyse de la sous-unité 28000 et par là-même l'apparition des formes activées.

\section{Commentaires et conclusion.}

Les interactions phospholipides membranaires-calpaine II ont été considérées par certains auteurs (Suzuki, 1987 ; Coolican et al., 1986) comme une étape importante de l'activation de cette protéase par autolyse en présence de calcium, cette étape conduisant à des formes enzymatiques nouvelles dont le seuil optimum en calcium s'avère beaucoup plus faible (Mellgren et al., 1982 ; Suzuki et al., 1981).

Les résultats obtenus dans nos conditions expérimentales mettent en évidence qu'en absence de substrat, les formes enzymatiques activées $80000 / 18000$ apparaissent beaucoup plus rapidement lorsque l'on est en présence de phospholipides acides tels que le phosphatidyl inositol ou la phosphatidyl sérine. Aucune différence significative par rapport au témoin n'a pu être notée en présence de phospholipides neutres (phosphatidyl choline).

En présence de substrat ( $\beta$-globine), bien que les formes 80 000/18 000 apparaissent plus rapidement en présence de PI et PS, I'hydrolyse de la sous-unité 28000 est cependant ralentie par rapport aux résultats obtenus sans substrat.

L'effet substrat induit sur le complexe PS-calpaine II une dégradation simultanée des deux sous-unités, phénomène non encore observé jusqu'alors. L'autolyse de la sous-unité 28000 reste cependant plus rapide en présence de $\mathrm{PI}$. Au vu des résultats obtenus, en présence de substrat et de $\mathrm{Ca}^{2+}$, l'incidence du PS sur la calpaine II semble être très spécifique.

Des études basées sur la caractérisation du type d'interactions calpaine II-phospholipides ainsi que les facteurs influant sur la dissociation de ces complexes après apparition des formes activées sont en cours.

$13^{e}$ Réunion du groupe Développement I.N.R.A. Cap d'Agde, 25-27 mai 1987.

\section{Références}

AZANZA J. L., RAYMOND J., ROBIN J. M., COTTIN P., DUCASTAING A., 1979. Purification and some physico-chemical and enzymic properties of a calcium-ion-activated neutral proteinase from rabbit skeletal muscle. J. biol. Chem., 183, 339-347. 
AZANZA J. L., RAYMOND J., ROBIN J. M., COTTIN P., DUCASTAING A., 1980. Proteinase neutre calcium dépendante du muscle squelettique de lapin : activité sur les protéines myofibrillaires. Biochimie, 62, 481-486.

BRADFORD M. H., 1976. A rapid and sensitive method for the quantitation of microgram quantities of protein utilizing the principle of dye binding. Anal. Biochem., 72, 248-254.

COOLICAN S. A., HAIECH J., HATHAWAY D. R., 1986. The role of subunit autolysis in activation of smooth muscle $\mathrm{Ca}^{2+}$-dependent proteases. J. biol. Chem., 261, 9, 4170-4176.

COOLICAN S. A., HATHAWAY D. R., 1984. Effect of L-a-phosphatidyl inositol on a vascular smooth muscle $\mathrm{Ca}^{2+}$-dependent protease : reduction of the $\mathrm{Ca}^{2+}$-requirement for autolysis. J. biol. Chem., 259, 11627-11630.

DAYTON W. R., GOLL D. E., ZEECE M. G., ROBSON M. R., REVILLE W. J., 1976a. A Ca ${ }^{2+}$ activated protease possibly involved in myofibrillar protein turnover. Purification from porcine muscle. Biochemistry, 15, 2150-2158.

DAYTON W. R., REVILLE W. J., GOLL D. E., STOMER M. H., 1976b. A calcium-activated protease involved in myofibrillar protein turnover. Partial characterization of the purified enzyme. Biochemistry, 15, 2159-2167.

De MARTINO G. N., HUFF A. N., CROALL D. E., 1986. Autoproteolysis of the small subunit of calcium-dependent protease $I I$ activates and regulates activity. J. biol. Chem., 261, $12047-12052$.

GARRET C., COTTIN P., DUFOURCO J., DUCASTAING A., 1988. Evidence for a $\mathrm{Ca}^{2+}$. independent association between calpain II and phospholipid vesicles. FEBS Letter, 227. 209-214.

HATHAWAY D. R., WERTHE D. K., HAERBELE J. R., 1982. Limited autolysis reduces the $\mathrm{Ca}^{2+}$ requirement of a smooth muscle $\mathrm{Ca}^{2+}$ activated protease. J. biol. Chem., 257, 9072-9077.

HUTSON R. G., KREBS E. G., 1968. Activation of skeletal muscle phosphorylase kinase by $\mathrm{Ca}^{2+}$. Identification of the kinase activating factor as a proteolytic enzyme. Biochemistry, 7 , 2116-2122.

IMAJOH S., KAWASAKI M., SUZUKI K., 1986. The amino terminal hydrophobic region of the small subunit of calcium activated neutral protease (CANP) is essential for its activation by phosphatidyl inositol. Biochem. J., 99, $1281-1284$.

LAEMMLI U. K., 1970. Cleavage of structural proteins during the assembly of the head of the bacteriophage T4. Nature, 227, 660-685.

MELLGREN R. L., REPETTE A., MUCK T. C., EASLY J., 1982. Rabbit skeletal muscle calciumdependent autoproteolysis. J. biol. Chem., 57, 7203-7209.

MERDACI N., COTTIN P., OUALI A., OBLED A., VALIN C., DUCASTAING A., 1983. Comparative effects of two calcium-activated neutral proteinases on ATPases activities. Relationship with ageing phenomenon. Sci. Aliments, 3, 499-512.

MORGAN M. E., CHUA B., BEINLICH C. J., 1980. Regulation of protein degradation in heart, 87-109. In WILDENTHAL, Degradation processes in heart and skeletal muscle. Elsevier/North-Holland Biomedical Press, Amsterdam.

PENNY I. F., TAYLOR M. A., HARRIS A. G., ETHERINGTON D. J., 1985. Purification and immunological characterisation of two calcium-activated neutral proteinases from rabbit skeletal muscle. Biochim. Biophys. Acta, 829, 244-252.

PUCA G. A., NOLA E., SICA V., BRESCIANI F., 1977. Estrogen binding proteins of calf uterus. Molecular and functional characterization of the receptor transforming factor: a calcium activated neutral protease. J. biol. Chem., 252, 1358-1366.

SUZUKI K., 1987. Calcium activated neutral protease: domain structure and activity regulation. Trends biochem. Sci., 12, 103-105.

SUZUKI K., TSUJI S., KUBOTA S., KIMURA Y., IMAHORI K., 1981. Limited autolysis of $\mathrm{Ca}^{2+}$-activated neutral protease (CANP) changes its sensitivity to $\mathrm{Ca}^{2+}$ ions. J. Biochem., 90, 275-278. 